\title{
Adoção de prontuário eletrônico do paciente em hospitais universitários de Brasil e Espanha. A percepção de profissionais de saúde*
}

\author{
Josivania Silva Farias** \\ Tomas de Aquino Guimaraes*** \\ Eduardo Raupp de Vargas**** \\ Pedro Henrique Melo Albuquerque*****
}

S umário: 1. Introdução e discussão teórica; 2. Método; 3. Resultados; 4. Discussão e conclusão.

Summary: 1. Introduction and theoretical discussion; 2. Method; 3. Results; 4. Discussion and conclusion.

\footnotetext{
* Artigo recebido em dez. 2010 e aceito em maio 2011. Agradecemos à Fundação Coordenação de Aperfeiçoamento de Pessoal de Nível Superior (Capes) pela bolsa PDEE (doutorado sanduíche) concedida ao primeiro autor. Agradecemos também às organizações hospitalares: Hospital de Clínicas de Porto Alegre e Hospital Universitário de Brasília, Brasil, e Hospital Universitario de Fuenlabrada, Espanha, por abrir espaço à coleta de dados, viabilizando a realização deste trabalho.

** Professora adjunta da Universidade de Brasília (UnB), Departamento de Administração. Doutora em administração pela UnB. Endereço: Universidade de Brasília (UnB). Campus Darcy Ribeiro, Departamento de Administração (ADM/Face), ICC Ala Norte, B1-576 - Asa Norte - CEP 70910900, Brasília, DF, Brasil. E-mail: josivania@unb.br.

*** Professor titular da UnB, Departamento de Administração. Doutor em sociologia pela Universidade de São Paulo (USP). Pesquisador CNPq, nível 1C. Endereço: Universidade de Brasília (UnB). Campus Darcy Ribeiro, Departamento de Administração (ADM/Face), ICC Ala Norte, B1-576 — Asa Norte — CEP 70910-900, Brasília, DF, Brasil. E-mail: tomas.aquino.guimaraes@ gmail.com.

**** Professor adjunto da UnB, Departamento de Administração. Doutor em administração pela Universidade Federal do Rio Grande do Sul (UFRGS). Endereço: Universidade de Brasília (UnB). Campus Darcy Ribeiro, Departamento de Administração (ADM/Face), ICC Ala Norte, B1-576 — Asa Norte — CEP 70910-900, Brasília, DF, Brasil. E-mail: ervargas@unb.br.

***** Professor assistente da UnB, Departamento de Administração. Mestre em estatística pela Universidade Federal de Minas Gerais (UFMG). Endereço: Universidade de Brasília (UnB). Campus Darcy Ribeiro, Departamento de Administração (ADM/Face), ICC Ala Norte, B1-576 — Asa Norte _ CEP 70910-900, Brasília, DF, Brasil. E-mail: pedro.melo.albuquerque@gmail.com.
} 
Palavras-chave: sistemas computadorizados de registros médicos; difusão de inovação; administração hospitalar.

KeY WORDs: medical records systems computerized; diffusion of innovation; hospital management.

O artigo descreve a percepção de profissionais de saúde a respeito do prontuário eletrônico do paciente na gestão de hospitais e o impacto dessa tecnologia no trabalho. A coleta de dados ocorreu em 2009 e 2010 com 336 usuários deste prontuário, em dois hospitais universitários, sendo um no Brasil e outro na Espanha. Os participantes da pesquisa preencheram um questionário contendo respostas padronizadas em escala de concordância do tipo Likert. Foram realizadas análises estatísticas descritivas e inferenciais e os resultados mostram que: a) esta tecnologia é percebida como um mecanismo que contribui com a gestão e a assistência hospitalar mediante mecanismos de controle; b) os respondentes com maior domínio de informática concordam mais do que aqueles com menor domínio a respeito do impacto desse prontuário no respectivo desempenho; c) há diferenças estatisticamente significativas nas médias de percepção do papel desse prontuário no desempenho quando considerado o tempo de serviço e o hospital (ou o país) onde o participante da pesquisa trabalha. Estudos adicionais são sugeridos no final.

Adoption of electronic patient record in teaching hospitals in Brazil and Spain. The perception of health professionals

This paper describes health professionals' perception upon electronic patient record on hospitals management and the impact of that technology on work. Data collection was undertook from 2009 to 2010 involving 336 users of this health record in two teaching hospitals, being one in Brazil and the other in Spain. The research respondents filled in a questionnaire with standardized answers on a Likert concordance scale. Descriptive and inferential statistics analysis were done and the main results show that: a) this technology is perceived as a mechanism of support of hospital management control; b) the more the respondents skills on managing information technology tools the more they agree about the record impact on their performance; c) there are significant statistically differences on average respondents perception upon the role of that record over their performance, taking into account length of service and the hospital (or country) where the research participants work. At the end further studies are suggested.

\section{Introdução e discussão teórica}

Hospitais são organizações integradas à economia de serviços e com o avanço da sociedade da informação utilizam, cada vez mais intensamente, tecnologias de informação e comunicação (TIC) na gestão de seus processos de trabalho 
e nas atividades inerentes à relação entre provedor e usuário na produção e oferta do serviço hospitalar. O prontuário eletrônico do paciente (PEP) é uma das inovações que têm sido adotadas por hospitais, como parte desse movimento de introdução de TIC, com a finalidade de obter ganhos de eficiência e de eficácia na gestão dessas organizações.

O levantamento realizado por Menachemi e colaboradores (2007) em 98 hospitais da Flórida discutiu a articulação entre segurança do paciente e emprego de tecnologias de informação na saúde. Utilizando base de dados da Healthcare Information and Management Systems Society (HIMSS), que realiza levantamentos anuais, cujas amostras variam de 2 mil a 5 mil hospitais, os resultados de regressões multivariadas revelaram que há importantes evidências de relação entre adoção de tecnologias de informação e segurança do paciente. Furukawa e colaboradores (2008) realizaram estudos com o mesmo banco de dados relativo às 98 organizações hospitalares na Flórida e concluíram que tamanho do hospital, nível de conhecimento, sistemas de parcerias, nível de "acreditação" do hospital, existência de fontes financiadoras são fatores associados à adoção de tecnologias de informação na saúde. Também descobriram que o tipo de tecnologia adotada poderá variar de uma região geográfica para outra.

Coye, Haselkorn e DeMello (2009) discutem em revisão de literatura sobre estudos realizados a respeito de sistemas de cuidado remoto do paciente (Remote Patient Management - RPM) que a adoção desses sistemas propicia à área de saúde dois ganhos importantes: reduz custos e reorganiza o processo de atenção ao paciente.

Perez e Zwicker (2010:197) estudaram fatores que afetam a adoção de prontuário eletrônico do paciente buscando conhecer os reflexos do uso desse prontuário para profissionais de saúde - usuários do sistema. Com resultados encontrados por meio de análise de equações estruturais e regressão linear múltipla, o levantamento realizado com 73 usuários em uma instituição de saúde brasileira permitiu descobrir que as influências são oriundas das características percebidas na inovação implantada. Estas características são: a) vantagem relativa - grau em que uma inovação é percebida como melhor que sua precursora; b) compatibilidade — grau em que uma inovação é percebida como consistente com valores, necessidade e experiências dos adotantes potenciais; e c) demonstração de resultado - grau em que os resultados do uso de uma inovação são tangíveis. Tudo isso, segundo o estudo, acarreta uma perspectiva de inovação no trabalho, sendo o PEP "um mediador de sucesso".

O prontuário eletrônico do paciente reorganiza dados clínicos e hospitalares e permite sua guarda em repositórios eletrônicos (Costa, 2001); con- 
tribui com a melhoria da redação da prescrição médica e da administração de medicamentos no horário prescrito (Gimenes et al., 2009) e permite a avaliação mais apurada das informações contidas no prontuário do paciente (Silva e Tavares-Neto, 2007), impondo a hospitais a reinterpretação de seus processos e métodos de trabalho (Perez e Zwicker, 2010). Pressupõe-se que o PEP seja uma inovação tecnológica e, em decorrência de sua adoção, surgem outras inovações que podem ser consideradas administrativas. Nessa linha, é importante identificar como a adoção do PEP é percebida por profissionais de saúde que o utilizam, bem como analisar impactos desse sistema de prontuário no trabalho desses profissionais.

Rogers (1995), ao propor uma teoria da difusão de inovações, cita cinco etapas correspondentes ao processo de difusão que podem ser cruciais para seu êxito ou fracasso: 1) conhecimento; 2) persuasão; 3) decisão; 4) implantação; e 5) confirmação. No estágio de decisão, os grupos pertencentes à organização poderão manifestar aceitação ou rejeição à inovação. Para o autor, "quando novas ideias são inventadas, difundidas e são adotadas ou rejeitadas, levando a certas consequências; então uma mudança social ocorre" (Rogers, 1995:6). Isso reforça que a adoção de uma inovação é um processo sociotécnico, em que mudanças tecnológicas resultam de processos sociais e dependem de novos comportamentos dos diversos atores sociais envolvidos.

Para Djellal e Gallouj (2005:829), a "trajetória informacional" tem se tornado cada vez mais evidente na gestão de fluxos de materiais, nos serviços de telemedicina, nos códigos de barras de medicamentos ou nas câmeras filmadoras úteis na detecção de doenças, entre outros. A implantação de redes e a produção da informação geram espaço para novas maneiras de usar a informação. Assim, torna-se possível gerenciar melhor os fluxos de informação em hospitais.

Como uma combinação sociotécnica, o planejamento da adoção de tecnologias da informação e comunicação no trabalho deve levar em conta como os indivíduos percebem fatores motivadores advindos do emprego dessas tecnologias em suas tarefas. A utilidade e a facilidade de uso de TIC interferem na crença do indivíduo de que um sistema de informação melhorará seu desempenho no trabalho e isso o levará a fazer uso do sistema. Exemplos disso seriam: qualidade do trabalho, rapidez na execução das tarefas, melhoria da produtividade, facilidade do uso da tecnologia e do uso de computadores na execução de tarefas, facilidade de apoio ao trabalho gerencial etc. (Davis, 1986; Dias, 2000).

Nessa linha, esta pesquisa teve como objetivo identificar a percepção de profissionais de saúde que atuam em dois hospitais universitários públicos, 
um do Brasil e um da Espanha, sobre o significado do prontuário eletrônico do paciente (PEP) e como este impacta o trabalho desses profissionais. Os dois países guardam similaridades na forma como seus sistemas nacionais de saúde foram desenhados e como são gerenciados. A pesquisa teve como pressupostos: a) que características biográficas e profissionais de usuários podem influenciar na percepção de que o PEP melhora seu desempenho individual; e b) que, na comparação de grupos independentes, há diferenças significativas a serem consideradas quanto à forma como usuários percebem o prontuário eletrônico.

\section{Método}

A pesquisa foi realizada no período de abril de 2009 a maio de 2010, considerando-se, inicialmente, três hospitais, e contou com uma fase de entrevistas e uma de aplicação de questionários. Na primeira fase foram entrevistados, com o suporte de um roteiro estruturado, sete gestores do Hospital Universitário de Brasília (HUB), Brasil, organização que empreendeu esforços, desde 2004, para implantar o PEP. Os resultados das entrevistas permitiram a elaboração de um questionário utilizado na segunda fase da pesquisa, realizada no Hospital de Clínicas de Porto Alegre (HCPA), Brasil, e no Hospital Universitário de Fuenlabrada (HUF), Espanha.

O HCPA foi criado em 1971, possui 786 leitos e é uma organização pública de direito privado. Esta organização integra uma rede de 44 hospitais universitários públicos brasileiros e é vinculada à Universidade Federal do Rio Grande do Sul (Hospital de Clínicas de Porto Alegre, 2010). Este hospital, desde a década de 1980, desenvolve automação de processos. O PEP do HCPA passou a ser implantado e a integrar diversas áreas do hospital a partir de meados da década de 1990 e é ferramenta em constante desenvolvimento para o aperfeiçoamento de seus aplicativos até o presente (2010).

O HUF iniciou suas atividades em 2004, tem 406 leitos e é um hospital público também submetido a tratamento jurídico de direito privado, estabelecido na Comunidade Autônoma de Madrid, vinculado à Universidad Rey Juan Carlos (Hospital Universitario de Fuenlabrada, 2008). Desde sua inauguração o HUF conta com PEP, que funciona de modo integrado com as áreas clínicas e administrativas do hospital.

O questionário utilizado na coleta de dados foi composto por 42 itens, na forma de afirmativas em sentido positivo. Este instrumento foi construído com base na revisão de literatura (Sittig, Kuperman e Fiskio, 1999:404; 
Massad, Marin e Azevedo Neto, 2003:7-9) e na análise de conteúdo de cinco entrevistas com gestores do HUB. Identificou-se que o prontuário eletrônico do paciente (PEP) atua como ator-rede mediador de uma relação baseada no controle de gestão e da assistência hospitalar. O controle de quatro dimensões (tempo, trabalho, custos e informação) confere ao PEP o papel de mediador da relação entre dois atores humanos: o gestor hospitalar e o usuário do sistema PEP.

O quadro 1 elucida um item relativo a cada dimensão para demonstrar o processo de construção do instrumento, adotando-se a escala tipo Likert de concordância de cinco alternativas como a estratégia de medida mais adequada (Günther, 1999).

$$
\text { Quadro } 1
$$

\section{Construto, dimensões, definição operacional e escala utilizados no instrumento de coleta de dados}

\begin{tabular}{|c|c|c|c|}
\hline Construto & Dimensões & $\begin{array}{l}\text { Definição operacional das } \\
\text { dimensões em itens }\end{array}$ & $\begin{array}{c}\text { Escala de mensuração } \\
\text { Likert }\end{array}$ \\
\hline \multirow{4}{*}{$\begin{array}{l}\text { PEP como } \\
\text { ator mediador } \\
\text { da gestão, } \\
\text { contribuindo } \\
\text { com o controle } \\
\text { gerencial e } \\
\text { assistencial }\end{array}$} & Controle de Tempo & $\begin{array}{l}\text { O prontuário eletrônico permite } \\
\text { acesso rápido às informações a } \\
\text { respeito do paciente }\end{array}$ & \multirow{4}{*}{$\begin{array}{l}\text { O respondente assinala, em } \\
\text { uma escala de cinco níveis, } \\
\text { seu grau de concordância } \\
\text { com o que está proposto } \\
\text { no item apresentado. } \\
\text { Escala: } \\
\text { (1) Discordo totalmente } \\
\text { (2) Discordo pouco } \\
\text { (3) Nem discordo, nem } \\
\text { concordo } \\
\text { (4) Concordo pouco } \\
\text { (5) Concordo totalmente }\end{array}$} \\
\hline & $\begin{array}{l}\text { Controle de } \\
\text { Trabalho }\end{array}$ & $\begin{array}{l}\text { O prontuário eletrônico contribui } \\
\text { para a padronização das rotinas } \\
\text { do hospital }\end{array}$ & \\
\hline & Controle de Custos & $\begin{array}{l}\text { O prontuário eletrônico permite } \\
\text { melhor planejamento de gastos } \\
\text { da assistência hospitalar }\end{array}$ & \\
\hline & $\begin{array}{l}\text { Controle de } \\
\text { Informação }\end{array}$ & $\begin{array}{l}\text { O prontuário eletrônico preserva } \\
\text { o sigilo das informações do } \\
\text { paciente }\end{array}$ & \\
\hline
\end{tabular}

Fonte: Elaborado pelos pesquisadores.

Os itens do questionário foram submetidos à análise de juízes, com a finalidade de validação teórica e de conteúdo. Foram realizadas duas rodadas de análise. Na primeira, os itens foram analisados por cinco juízes; na segunda rodada, seis juízes, incluídos os cinco que atuaram na primeira rodada, procederam à nova análise dos itens. Quatro itens alocados inicialmente na dimensão Controle de Informação foram deslocados para a dimensão Controle de Trabalho, dado que mais de $80 \%$ dos juízes apontaram nessa direção. Além 
desses 42 itens, o questionário continha uma seção destinada a dados biográficos e profissionais dos respondentes.

A tradução do instrumento do português para o espanhol contou com a análise de um profissional do HUF, de nacionalidade espanhola, com pós-graduação em economia da saúde, que corrigiu e adaptou o texto para a língua espanhola.

Foram distribuídos 2.302 questionários, sendo 1.592 no HCPA e 710 no HUF, que corresponde à população de profissionais de saúde que utilizam o prontuário eletrônico nesses hospitais. O questionário foi distribuído a essa população acompanhado de uma correspondência onde se explicitavam os objetivos da pesquisa, o fato de que o hospital havia autorizado sua realização e a garantia de anonimato. Sua distribuição ocorreu de duas formas: no HCPA, Brasil, por meio eletrônico, via hiperlink enviado por e-mail, e no HUF, Espanha, em meio impresso. Cada participante deveria assinalar seu nível de concordância a respeito dos itens do questionário utilizando uma escala do tipo Likert, de cinco pontos: 1: discordo totalmente, 2: discordo pouco, 3: nem concordo nem discordo, 4: concordo pouco e 5: concordo totalmente.

Dos 2.302 questionários distribuídos retornaram 362, sendo excluídos 26 , respondidos por pessoas que não faziam parte da população do estudo, restando 336 questionários válidos, correspondentes a 15\% do total. Destes 336 questionários, 178 eram do HCPA (11\% de taxa de retorno) e 158 do HUF ( $22 \%$ de taxa de retorno). Essas taxas de retorno são adequadas para pesquisas desta natureza, como sugerem Skitka e Sargis (2006) e Hill e Hill (2002).

Constatou-se que houve 18 casos omissos, 5,36\% do total. Decidiu-se por manter esses casos sem informação, uma vez que o prejuízo para a análise é pequeno em relação ao ganho em manter a observação (em termos de tamanho amostral), pois as observações perdidas não chegam a reduzir a eficiência da avaliação ou estimação do fenômeno estudado, como sugere Rubin (2004).

Quanto à análise de casos extremos (outliers), considerando uma distribuição normal, espera-se que 5\% das observações estejam acima (ou abaixo) de dois desvios-padrão. Com base nisto, foram realizados os procedimentos: a variável que sintetiza a solução fatorial foi analisada via Box Plot, constatandose 11 observações extremas. Uma vez que esse número está abaixo do limite mínimo aceitável, optou-se por não excluí-lo da análise, novamente considerando-se o argumento do aumento do tamanho da amostra. Para Murteira (1993:100), “a identificação quase certa de outliers só é em geral possível para amostras com 500 ou mais observações" - o que não é o caso deste estudo, que registra de 309 a 336 observações por variável. Não foram identificados 
casos extremos nas variáveis relativas ao perfil dos respondentes. O quadro 2 mostra o perfil dos participantes da pesquisa.

\section{Dados dos participantes da pesquisa}

\begin{tabular}{|c|c|c|}
\hline $\mathrm{N}=336$ sujeitos & $\begin{array}{l}\text { Tempo de serviço nos hospitais } \\
\text { do estudo: } \\
\text { Média: } 6,7 \text { anos D.p }=3,7 \text { anos } \\
\text { HUF: } 65,6 \% \text { entre } 4 \text { e } 5 \text { anos } \\
\text { HCPA: } 65,2 \% \text { com } 10 \text { anos ou } \\
\text { mais }\end{array}$ & $\begin{array}{l}\text { Tempo de uso do PEP: } \\
\text { Média: } 5,8 \text { anos D.p=2,9 anos } \\
\text { Mínima }=\text { menos de } 1 \text { ano; Máxima } \\
=10 \text { anos } \\
\text { HUF: } 60 \% \text { entre } 5 \text { e } 6 \text { anos } \\
\text { HCPA: } 76,3 \% \text { de } 0 \text { a } 9 \text { anos e } 23,7 \% \\
\text { acima de } 10 \text { anos }\end{array}$ \\
\hline $\begin{array}{c}\text { Sexo: } \\
\text { 68\% Feminino } \\
\text { 32\% Masculino } \\
\text { HUF: 69,6\% Feminino e } \\
\text { 30,4\% Masculino } \\
\text { HCPA: 66,7\% Feminino e } \\
\text { 33,3\% Masculino }\end{array}$ & \multicolumn{2}{|c|}{$\begin{array}{l}\text { Idade: } \\
\text { Média: } 40,5 \text { anos D.p }=10,6 \text { anos } \\
\text { Mínima: } 21 \text { anos; Máxima: } 67 \text { anos } \\
\text { HUF: } 68,5 \% \text { entre } 28 \text { e } 40 \text { anos } \\
\text { HCPA: } 63,4 \% \text { entre } 40 \text { e } 60 \text { anos }\end{array}$} \\
\hline $\begin{array}{l}\text { Hospital de vínculo: } \\
\text { Hospital de Fuenlabrada Madrid } \\
\text { (HUF): } 158 \text { sujeitos }(47 \%) \\
\text { Hospital de Clínicas de Porto } \\
\text { Alegre (HCPA): } 178 \text { sujeitos } \\
(53 \%)\end{array}$ & $\begin{array}{l}\text { Cargo/Função ocupada: } \\
\text { 49\% Médico } \\
\text { 48\% Enfermeiro } \\
\text { 3\% Profissional de saúde de outra } \\
\text { especialidade } \\
\text { HUF: 33,5\% Médicos, 65,8\% } \\
\text { Enfermeiros, 0,7\% Outros. } \\
\text { HCPA: 63,5\% Médicos, 32\% } \\
\text { Enfermeiros, 4,5\% Outros. }\end{array}$ & $\begin{array}{l}\text { Tipo de vínculo com os hospitais } \\
\text { do estudo: } \\
\text { 66\% Funcionário Efetivo } \\
\text { 15\% Empregado Temporário } \\
\text { 19\% outras situações (p. ex.: profes- } \\
\text { sores de faculdade de medicina que } \\
\text { lecionam utilizando o PEP) } \\
\text { HUF: } 67,5 \% \text { Funcionário Efetivo } \\
\text { HCPA: } 64,7 \% \text { Funcionário Efetivo }\end{array}$ \\
\hline
\end{tabular}

Fonte: Dados da pesquisa.

Para a análise dos dados foram utilizados o SPSS 15.0 e o SAS 9.2.

\section{Resultados}

Como mostra o quadro 2, o perfil dos respondentes de ambos os hospitais é assemelhado no que se refere às variáveis sexo e tipo de vínculo. No tocante ao cargo/função ocupada, o HUF possui mais enfermeiros do que médicos, enquanto o HCPA possui mais médicos do que enfermeiros participando da 
pesquisa. Procurou-se conhecer a distribuição dessa amostra no universo para verificar se isso reflete a população como um todo, nos dois hospitais, o que se comenta a seguir.

No HUF, a população de enfermeiros corresponde a 55\% dos profissionais de saúde com nível superior e 41\% são médicos; 4\% são de outras especialidades. No HCPA, 65\% são médicos, 30\% são enfermeiros e 5\% estão distribuídos em outras especialidades. Portanto, a amostra da pesquisa possui características muito semelhantes às do universo de profissionais de saúde que trabalham nos dois hospitais da pesquisa.

Destaca-se o fato de que os participantes da pesquisa do HUF são mais jovens (68,5\% estão com idade entre 28 e 40 anos) do que os participantes do HCPA (63,4\% estão entre 40 e 60 anos). Uma possível explicação para esses dados é o fato de que o HUF existe há seis anos (2004-10), já o HCPA existe há quase 40 anos (1972-2010).

Quanto à participação dos respondentes no desenvolvimento e na implantação do prontuário eletrônico do paciente (PEP) nos dois hospitais, ficou constatado que $61 \%$ dos respondentes não participaram desse processo, $26 \%$ participaram da implantação, embora não tenham participado das discussões de desenvolvimento da tecnologia, e 13\% participaram tanto do desenvolvimento como da implantação do PEP. No HUF, 55,3\% dos usuários não participaram das discussões, tampouco da implantação do PEP. Este percentual sobe para $60,9 \%$ no HCPA. Quanto ao percentual dos que não participaram do desenvolvimento, porém participaram da implantação do PEP, estes correspondem a $30,9 \%$ no HUF e a $26,1 \%$ no HCPA. Por fim, considerando-se os que participaram tanto do desenvolvimento como da implantação do PEP, os usuários correspondem a 13,8\% no HUF e a 13\% no HCPA. Isto mostra uma situação parecida quanto ao nível de envolvimento dos sujeitos com o PEP nos dois hospitais da pesquisa.

Identificou-se, ainda, a autoanálise dos respondentes sobre seu domínio de informática, considerando-se os dois hospitais (HUF e HCPA), e foi constatado que $20 \%$ dos indivíduos julgavam ter ótimo domínio de informática, $59 \%$ julgavam ter bom domínio, 20\% consideravam ter domínio regular e 1\% avaliavam seu domínio de informática como ruim ou péssimo. Analisando-se em separado, no HUF, Espanha, 79,8\% dos respondentes afirmaram que seu domínio está entre os níveis "bom" ou "ótimo". Este contingente é 1,7 ponto percentual menor no HCPA, Brasil, onde 78,1\% dos usuários de PEP afirmaram que seu domínio de informática é "bom" ou "ótimo". A percepção que o usuário de PEP tem sobre seu domínio de informática mostrou-se como variável relevante no modelo de regressão que será apresentado adiante. 
Procedeu-se, em seguida, à análise fatorial exploratória (AFE) com o objetivo de verificar a consistência interna do instrumento de coleta. A análise exploratória das observações forneceu um coeficiente de Kaiser-Mayer-Olkin (KMO) de 0.939 e teste de esfericidade de Bartlett apresentando Sig. $=0.000$ (Chi-Square aprox. $=4523.151 ; d f=253$ ). Portanto, verificou-se adequação dos dados para proceder à AFE (Tabachnick e Fidell, 2001). Fixou-se para a realização da AFE carga de contribuição da variável ao componente fatorial, ser $\geq .30$ e apresentar comunalidade $\left(\mathrm{h}^{2}\right) \geq .50$.

A análise com o método dos componentes principais e rotação varimax indicou a estimativa do número possível de fatores a partir de autovalores iguais ou superiores a 1,00. Analisando-se a variância total explicada, e procedendo-se à extração de fatores por meio do critério de análise visual do diagrama de raízes latentes (Scree Plot) e da carga de contribuição de cada variável aos componentes fatoriais observada na matriz de componentes, verificou-se que três componentes reuniam 23 variáveis e esse conjunto totalizava $61,45 \%$ da variância total explicada, com Alpha de Cronbach $\alpha$ bruto $=0.937$ e $\alpha$ padronizado $=0.939$. Os demais componentes apresentavam contribuições pequenas, em torno de $1 \%$ a 3\% de explicação, cujos autovalores mostravam-se inferiores a 1,00 .

Os resultados da AFE indicaram uma solução fatorial composta por três componentes que podem ser visualizados no Diagrama de Raízes Latentes (Scree Plot), gráfico a seguir.

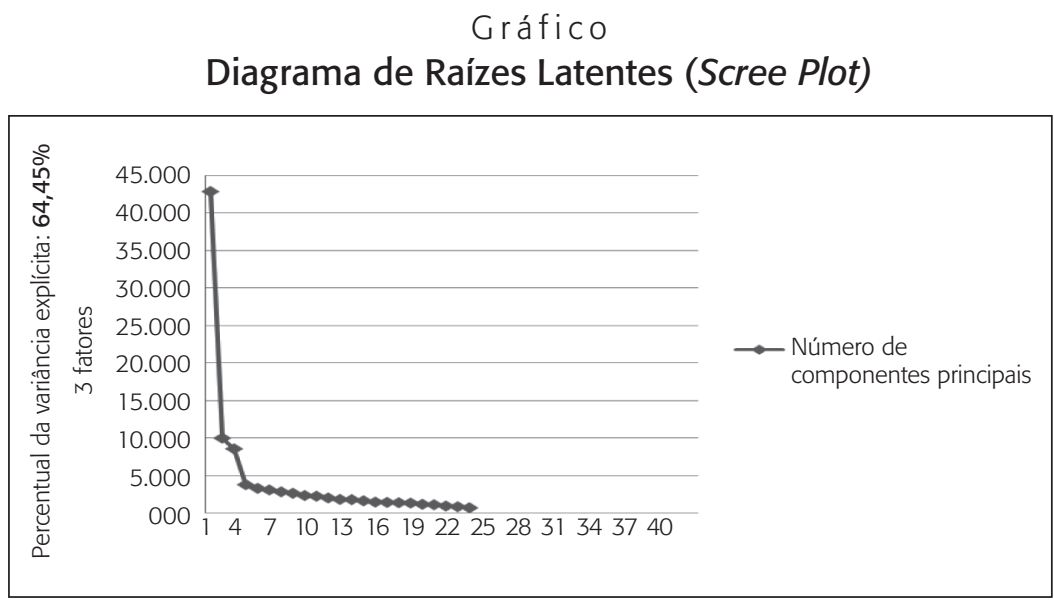

Fonte: Dados da pesquisa. 
A AFE possibilitou a geração de uma variável estatística a que se denominou "INDIC" (indicador), que permite obter resultados agrupados relativos à média e ao desvio padrão do conjunto de todas as variáveis.

Para verificar média e desvio padrão correspondentes à variável estatística gerada pelos componentes fatoriais verificados na AFE, procedeu-se à transformação linear em função do tipo de escala utilizada no instrumento de coleta. Ressalte-se que a transformação linear não altera as propriedades inerentes a cada índice analisado. O nível de concordância quanto ao indicador apresentou-se, inicialmente, como mínimo $=-2.30$ e máximo $=1.16$, centralizados em zero. Portanto, para clarificar a compreensão em escala com mínimo 1 e máximo 5, utilizada no questionário, procedeu-se à transformação linear, chegando-se aos seguintes valores: média geral do indicador que sintetiza o conjunto de variáveis que compõem os três fatores: 3,66 (d.p.=0,84). Para chegar a esta média ponderada foi necessário considerar o autovalor de cada um dos três fatores encontrados.

A tabela 1 apresenta um resumo da análise das variáveis da pesquisa, considerando médias e desvios padrão, além da contribuição de cada variável para a composição dos fatores com suas comunalidades $\left(\mathrm{h}^{2}\right)$ :

Tabela 1

Variáveis, cargas de contribuição aos fatores, comunalidades $\left(h^{2}\right)$, médias e desvios padrão

\begin{tabular}{|c|c|c|c|c|c|c|}
\hline № Variável & $\begin{array}{l}\text { FATOR } 1 \\
\text { Controle do } \\
\text { Trabalho }\end{array}$ & $\begin{array}{l}\text { FATOR } 2 \\
\text { Controle } \\
\text { de Custos }\end{array}$ & $\begin{array}{c}\text { FATOR } 3 \\
\text { Controle de } \\
\text { Informações }\end{array}$ & $\left(h^{2}\right)$ & Média & d.p. \\
\hline $\begin{array}{l}\text { V3 - A informação existente no } \\
\text { prontuário eletrônico é acessível } \\
\text { somente por pessoas autorizadas. }\end{array}$ & & & .800 & .809 & 3.83 & 1.27 \\
\hline $\begin{array}{l}\text { V4 - O prontuário eletrônico } \\
\text { permite que as informações do } \\
\text { paciente sejam armazenadas de } \\
\text { forma segura. }\end{array}$ & & & .822 & .795 & 3.96 & 1.10 \\
\hline $\begin{array}{l}\text { V7 - O prontuário eletrônico } \\
\text { preserva o sigilo das informações } \\
\text { do paciente. }\end{array}$ & & & .798 & .743 & 3.36 & 1.27 \\
\hline $\begin{array}{l}\text { V8 - O prontuário eletrônico } \\
\text { permite a guarda com segurança } \\
\text { das informações relativas à } \\
\text { assistência. }\end{array}$ & & & .804 & .829 & 3.79 & 1.17 \\
\hline
\end{tabular}

Continua 


\begin{tabular}{|c|c|c|c|c|c|c|}
\hline № Variável & $\begin{array}{l}\text { FATOR } 1 \\
\text { Controle do } \\
\text { Trabalho }\end{array}$ & $\begin{array}{l}\text { FATOR } 2 \\
\text { Controle } \\
\text { de Custos }\end{array}$ & $\begin{array}{l}\text { FATOR } 3 \\
\text { Controle de } \\
\text { Informações }\end{array}$ & $\left(h^{2}\right)$ & Média & d.p. \\
\hline $\begin{array}{l}\text { V9 - O uso do prontuário } \\
\text { eletrônico propicia uma maior } \\
\text { qualidade de informações às } \\
\text { pesquisas em um hospital } \\
\text { universitário. }\end{array}$ & .584 & & & .927 & 4.27 & .99 \\
\hline $\begin{array}{l}\text { V12 - O prontuário eletrônico } \\
\text { evita erros na transcrição de } \\
\text { nomes de medicamentos e } \\
\text { materiais. }\end{array}$ & & & .427 & .946 & 3.71 & 1.32 \\
\hline $\begin{array}{l}\text { V14 - A segurança do registro } \\
\text { de informações em prontuário } \\
\text { eletrônico é maior do que aquela } \\
\text { obtida em prontuário impresso. }\end{array}$ & & & .597 & .903 & 3.66 & 1.21 \\
\hline $\begin{array}{l}\text { V16 - O prontuário eletrônico } \\
\text { proporciona retorno positivo } \\
\text { do investimento realizado em } \\
\text { hospitais que o adotam. }\end{array}$ & & .597 & & .790 & 4.10 & .92 \\
\hline $\begin{array}{l}\text { V17 - Há economia de dinheiro } \\
\text { quando se emprega o prontuário } \\
\text { eletrônico em um hospital. }\end{array}$ & & .734 & & .826 & 3.93 & .94 \\
\hline $\begin{array}{l}\text { V18 - O prontuário eletrônico } \\
\text { permite o melhor planejamento } \\
\text { dos gastos da assistência } \\
\text { hospitalar. }\end{array}$ & & .819 & & .794 & 3.92 & .95 \\
\hline $\begin{array}{l}\text { V19 - O prontuário eletrônico } \\
\text { auxilia no controle de consumo de } \\
\text { recursos do hospital. }\end{array}$ & & .848 & & .844 & 3.99 & .97 \\
\hline $\begin{array}{l}\text { V20 - O prontuário eletrônico } \\
\text { contribui para a sustentabilidade } \\
\text { financeira de hospitais que o } \\
\text { utilizam. }\end{array}$ & & .859 & & .860 & 3.80 & .94 \\
\hline $\begin{array}{l}\text { V21 - O prontuário eletrônico } \\
\text { contribui para a eficiência (menor } \\
\text { gasto e maior benefício) da } \\
\text { assistência hospitalar. }\end{array}$ & & .815 & & .760 & 3.83 & .95 \\
\hline $\begin{array}{l}\text { V23 - O prontuário eletrônico } \\
\text { integra informaç̃̃es, promovendo } \\
\text { o andamento ágil da assistência. }\end{array}$ & .575 & & & .845 & 4.12 & .97 \\
\hline
\end{tabular}

Continua 


\begin{tabular}{|c|c|c|c|c|c|c|}
\hline № Variável & $\begin{array}{l}\text { FATOR } 1 \\
\text { Controle do } \\
\text { Trabalho }\end{array}$ & $\begin{array}{l}\text { FATOR } 2 \\
\text { Controle } \\
\text { de Custos }\end{array}$ & $\begin{array}{c}\text { FATOR } 3 \\
\text { Controle de } \\
\text { Informações }\end{array}$ & $\left(h^{2}\right)$ & Média & d.p. \\
\hline $\begin{array}{l}\text { V26 - O prontuário eletrônico } \\
\text { controla o ritmo do andamento da } \\
\text { assistência. }\end{array}$ & .601 & & & .780 & 3.74 & 1.08 \\
\hline $\begin{array}{l}\text { V27 - O prontuário eletrônico } \\
\text { agiliza prescrição na assistência ao } \\
\text { paciente. }\end{array}$ & .541 & & & .801 & 3.92 & 1.16 \\
\hline $\begin{array}{l}\text { V30 - O prontuário eletrônico } \\
\text { contribui para a qualidade da } \\
\text { assistência em hospitais. }\end{array}$ & .665 & & & .809 & 4.00 & 1.04 \\
\hline $\begin{array}{l}\text { V32 - O prontuário eletrônico } \\
\text { permite uma melhor formação } \\
\text { de alunos e residentes em um } \\
\text { hospital universitário. }\end{array}$ & .771 & & & .749 & 3.63 & 1.17 \\
\hline $\begin{array}{l}\text { V33 - O prontuário eletrônico } \\
\text { mostra o que está realmente } \\
\text { funcionando na assistência } \\
\text { hospitalar. }\end{array}$ & .687 & & & .883 & 3.44 & 1.13 \\
\hline $\begin{array}{l}\text { V37 - Meu desempenho na } \\
\text { assistência hospitalar é melhor } \\
\text { com o uso do prontuário } \\
\text { eletrônico. }\end{array}$ & .770 & & & .824 & 3.55 & 1.27 \\
\hline $\begin{array}{l}\text { V38 - O prontuário eletrônico } \\
\text { interfere de modo positivo } \\
\text { na minha relação funcional } \\
\text { (empregatícia) com este hospital. }\end{array}$ & .764 & & & .757 & 3.55 & 1.15 \\
\hline $\begin{array}{l}\text { V39 - Tenho maior controle } \\
\text { sobre meu trabalho ao utilizar o } \\
\text { prontuário eletrônico em minhas } \\
\text { atividades. }\end{array}$ & .791 & & & .801 & 3.75 & 1.17 \\
\hline $\begin{array}{l}\text { V41 - O prontuário eletrônico é } \\
\text { adequado às minhas tarefas neste } \\
\text { hospital. }\end{array}$ & .680 & & & .767 & 3.84 & 1.08 \\
\hline
\end{tabular}

Fonte: Dados da pesquisa.

O modelo teórico-empírico inicial pressupunha que o PEP se explicava a partir de quatro dimensões (controle de informação, controle de tempo, controle de custos e controle de trabalho). Após a verificação dos dados encontrados na AFE, três fatores se mantiveram: controle de trabalho, controle de custos e controle de informações. Algumas variáveis que descreviam o 
controle de tempo foram incorporadas no processo de rotação ao Fator 1 (controle de trabalho), o que é compreensível, uma vez que a organização e a melhoria de rotinas e processos de trabalho podem contribuir com a agilidade no processo assistencial, melhorando a qualidade geral do processo de trabalho.

Com a finalidade de verificar, especificamente, a percepção dos respondentes a respeito do impacto do PEP no trabalho que realizam, isolou-se o item 37 que continha a seguinte assertiva: "meu desempenho na assistência hospitalar é melhor com o uso do prontuário eletrônico". Este item foi transformado em variável dummy com dois níveis de resposta ( 0 ou 1) e considerada variável dependente. Atribuiu-se o valor zero para os pontos 1, 2 (discordância) e 3 (neutralidade) da escala, e valor 1 para os pontos 4 e 5 da escala, que sinalizavam concordância com a afirmativa. Após essa transformação realizou-se análise de regressão logística múltipla.

O conjunto de variáveis independentes (elegíveis para entrada na Stepwise) que compuseram o modelo da regressão logística correspondeu às variáveis biográficas e profissionais dos indivíduos e referem-se a: tempo como usuário de PEP; domínio de informática; sexo; idade; tempo de serviço no hospital; nível de participação na adoção do PEP (no desenvolvimento, na implantação, em nenhum dos dois estágios); categoria profissional (médico, enfermeiro, outra especialidade); tipo de vínculo (efetivo ou temporário), e hospital de vínculo (HUF ou HCPA).

Empregando-se o método Stepwise, foram obtidos três passos de ajuste do modelo de regressão, considerando 0.5 de significância de entrada das variáveis e limitando a não ultrapassar 0.05 de significância para que a variável se mantivesse no modelo, permanecendo no terceiro passo a variável: domínio de informática $(p=0.0170)$.

Na regressão logística, a análise da estimativa de máxima verossimilhança com a variável "domínio de informática" indicou, a um nível de significância $p<0.05$, que, se o indivíduo tem domínio de informática, a propensão em concordar com a assertiva de que o uso do PEP melhora seu desempenho no trabalho aumenta $(B=0.4018$, erro padrão 0.1701, Wald $=5.5789$, Sig. $=0.0182$ ). A análise da razão de chances (Odds Ratio - OR) também contribuiu para a compreensão do modelo, conforme interpretação que se pode fazer a partir da tabela 2 . 
Tabela 2

Estimativas da razão de chances (Odds Ratio)

\begin{tabular}{|cccc|}
\hline & Estimativa de & \multicolumn{2}{c|}{ Wald } \\
Efeito (Variável) & pontos & 95\% & Limite de Confiança \\
\hline Domínio de Informática & 1.495 & 1.013 & 2.086 \\
\hline
\end{tabular}

Fonte: Dados da pesquisa.

Para cada unidade aumentada na variável "domínio de informática" aumenta-se em $49,5 \%$ a chance de o usuário concordar com a variável 37 , que afirma que seu desempenho melhora com a utilização de prontuário eletrônico. Assim, o modelo de regressão pode ser descrito da seguinte forma:

Figura

Modelo de regressão logística

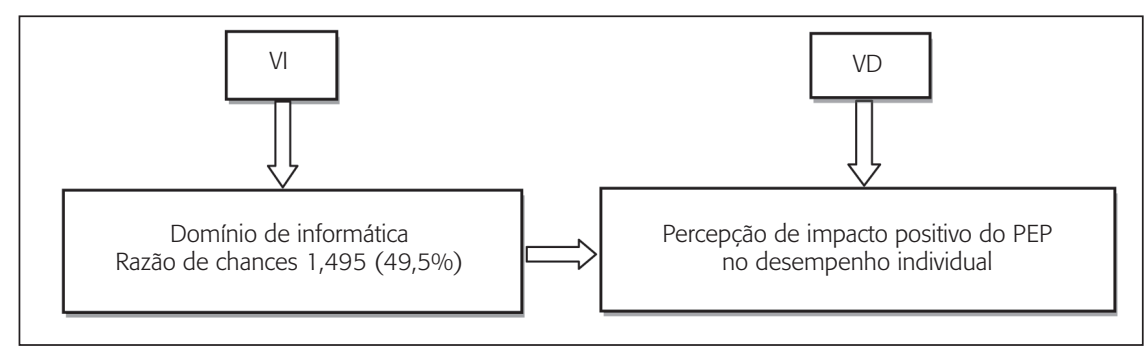

Fonte: Dados da pesquisa.

Foram analisados os pressupostos de adequação do modelo de regressão (Allison, 1999), sendo este considerado adequado. As probabilidades de respostas preditas e observadas mostram que o número de pares concordantes $(36,4 \%)$ é maior que o de pares discordantes (23\%); que o $p$-valor de avaliação de dependência dos resíduos foi igual a 0.4468 ( $p>0.05)$; que na avaliação de grupos há aproximação entre o total observado e o total esperado, para dummy 1 e dummy 0; que o teste de adequação de Hosmer e Lemeshow, com $p$-valor $=0.5580(p>0.05)$, Qui-Quadrado $=1.1669$ e 2 graus de liberdade $(d f)$, sugere a não rejeição da hipótese nula de boa adequação do modelo.

Considerando-se um nível de significância $p>0.05$, o teste KolmogorovSmirnov $(K S)$ apresentou Sig. $=0.046$, mostrando indícios de que a suposição de normalidade é fracamente rejeitada na distribuição dos dados, quando consideradas as 23 variáveis, após a AFE. Entretanto, para as 42 variáveis do instrumento de coleta, o $p$-valor representa Sig. $=0.085$, sendo a normalidade 
confirmada. Diante disto, realizou-se o teste $t$, porém, devido à proximidade do $p$-valor $=0.05$, ressalta-se que é inconclusivo por não apresentar robustez devido à ausência de normalidade. Adotou-se 95\% de intervalo de confiança e $5 \%$ de significância, sendo realizado o teste $t$ (Student) com variáveis de perfil dos indivíduos - nível de envolvimento no desenvolvimento e na implantação do prontuário eletrônico (ter participado ou não ter participado de desenvolvimento e implantação da tecnologia); tempo de uso do PEP (menor ou igual a cinco anos ou mais de cinco anos); nível de domínio de informática (abaixo de regular ou entre bom e ótimo); categoria profissional (médico; enfermeiro); sexo; idade; tipo de vínculo empregatício com o hospital (efetivo ou temporário); tempo de serviço no hospital (menor ou igual a cinco anos ou mais de cinco anos) e hospital/país (HUF, Espanha, ou HCPA, Brasil) a que pertence o respondente. $\mathrm{O}$ objetivo era identificar diferenças de médias que fossem significativas entre grupos independentes no tocante à percepção geral do prontuário eletrônico do paciente, admitindo-se na análise igualdade de variância assumida e igualdade de variância não assumida. Com isso, os pressupostos estabelecidos na introdução deste estudo poderiam ser verificados estatisticamente.

Duas variáveis apresentaram, no teste $t$ (Student), diferenças significativas de médias de percepção do PEP entre grupos, ao nível de 5\% $(p<0.05)$ : hospital/país a que pertence o respondente e tempo de serviço no hospital.

Verificou-se que as médias de percepção do PEP relativas a cada grupo corresponderam respectivamente a 3,63 (d.p. $=0.91)$ entre os respondentes do HCPA, Brasil, e 3,69 (d.p.= 0.75) entre os respondentes do HUF, Espanha, e que a um nível de significância $p<0.05($ Sig. $=0.004)$ há diferença de percepção do PEP entre estes grupos de usuários.

Foram também comparadas as médias de percepção do PEP segundo o tempo de serviço dos respondentes: profissionais com tempo menor ou igual a cinco anos, cuja média de concordância é 3,71 (d.p. $=0.77$ ) e profissionais com tempo de serviço maior que cinco anos, cuja média é 3,62 (d.p. $=0.89$ ). Constatou-se ao nível $p<0.05$ (Sig. $=0.018$ ) que havia diferença significativa de percepção do PEP entre os dois grupos.

\section{Discussão e conclusão}

A análise fatorial sugeriu uma solução fatorial composta por três fatores, sendo o PEP uma tecnologia composta por mecanismos de controle - de trabalho, de custos e de informação. 
A média de concordância com as assertivas que definem a variável estatística (Indicador) composta pelas variáveis resultantes da AFE (23 variáveis) corresponde a 3,66 (d.p. $=0,84)$, o que sinaliza para um nível de concordância moderado, por parte do usuário, em relação à percepção do prontuário eletrônico como uma tecnologia que contribui com a eficiência da gestão hospitalar.

Observa-se que o PEP pode ser classificado como uma inovação administrativa (Djellal e Gallouj, 2007; Birkinshaw, Hamel e Mol, 2008). Este tipo de inovação propicia reorganização administrativa e logística e, com a introdução de um sistema de prontuário eletrônico, outros sistemas que compõem a gestão hospitalar, por exemplo, controle de pacientes, de uso de medicamentos e custos, ganham mais rigor e correção, uma vez que as informações dos pacientes são registradas em um sistema de informações que integra as áreas assistencial e administrativa, com possibilidade de gerar indicadores de performance para essas áreas. Essas vantagens foram apontadas na literatura por Sittig, Kuperman e Fiskio (1999); Costa (2001); Massad, Marin e Azevedo Neto (2003).

Concernente ao Fator 1, controle do trabalho, a discussão sociotécnica envolvendo a percepção do usuário é fundamental em processos de adoção de inovação e isso enseja considerar, simetricamente, as inovações tecnológicas e não tecnológicas, em uma abordagem integradora (Gallouj, 2002; Djellal e Gallouj, 2001). A inovação de caráter tecnológico corresponde, neste estudo, à inserção da ferramenta de tecnologia de informação denominada prontuário eletrônico do paciente, que traz consigo modificações na gestão e no processo de organização do trabalho, o que resulta em uma inovação administrativa.

Esse prontuário também pode contribuir com a agilidade da assistência hospitalar, pois auxilia na integração de informações do paciente e agiliza o andamento da assistência e da prescrição ao paciente. Estas constatações são sustentadas pelas afirmativas redigidas no conjunto de variáveis de número 23, 26 e 27 constantes da tabela 1, com as quais o respondente manifestava seu grau de concordância. A média entre as variáveis oscilou entre 3,74 e 4,12, cujos valores ultrapassam o nível de discordância (alternativas 1 ou 2 da escala Likert) e de neutralidade (alternativa 3 da escala) com as afirmativas apresentadas. Gimenes e colaboradores (2009) destacam que o PEP contribui com a administração de medicamentos no horário prescrito, e para Costa (2001) esse prontuário reorganiza dados clínicos e hospitalares. Portanto, os achados são corroborados pela literatura.

A inovação é um fenômeno "multifacetado". Não envolve somente processos ou recursos, mas também ideias, transações, contexto, resultados e pes- 
soas (Van de Ven e Angle, 2000). A inovação pode modificar rotinas laborais, promovendo a inserção de novos métodos e processos. Nos hospitais estudados, o PEP é compreendido como uma inovação que contribui com a gestão da assistência, padronizando rotinas de trabalho, minimizando erros de prescrição ou ainda evitando a duplicação de tarefas.

O significado do prontuário eletrônico para o profissional de saúde se traduz no reconhecimento que este ator humano faz das funções daquele ator mediador não humano. O PEP apoia o processo de atenção à saúde, permite o registro seguro de ações médicas, apoia pesquisas, promove o ensino - as variáveis V9 e V32 incorporadas ao Fator 1 retratam este aspecto do PEP, de formação de alunos e contribuição com a pesquisa -, e o gerenciamento de serviços (Massad, Marin e Azevedo Neto, 2003:4), além de organizar rotinas de trabalho.

Essas atribuições do PEP o confirmam como ator mediador, que, segundo Latour (2000, 2001, 2004), é o ente componente da rede que, com a incumbência de mediar, não somente transporta, mas também transforma o que transporta, além de transformar comportamentos de outros componentes da rede em questão. No Fator 1 se verifica, ademais, que o PEP é percebido como ferramenta que contribui para a qualidade da assistência em hospitais (V30), para o controle do trabalho (V33, V39) e para a melhoria de desempenho de indivíduos (V37), bem como se ajusta e melhora as rotinas assistenciais (V41) e a relação do indivíduo com a organização hospitalar a que pertence (V38). Perez e Zwicker (2010) assinalam que o PEP impõe a reinterpretação de métodos e processos de trabalho, atuando como um mediador de sucesso.

No tocante ao controle de custos (Fator 2), a AFE permite verificar, nas variáveis V16 a V21 constantes da tabela 1, que a economia de dinheiro e a otimização no planejamento e utilização de recursos financeiros são ganhos proporcionados pelo emprego do PEP para a gestão hospitalar e assistencial. Assim, este prontuário auxilia no controle de recursos (V19), de modo a contribuir com a sustentabilidade financeira da organização hospitalar (V20). Esses resultados são compatíveis com os resultados de Coye, Haselkorn e DeMello (2009), que informam que sistemas de cuidado do paciente propiciam à área de saúde a redução de custos. Esta ferramenta "mostra como o dinheiro tem sido usado e o quê, de bom, foi realmente feito com ele..." (Massad, Marin e Azevedo Neto, 2003:2).

Com relação ao controle de informações (Fator 3), Djellal e Gallouj (2005) citam a melhoria da gestão de fluxos de informação como vantagem relativa ao emprego de tecnologias de informação e comunicação na gestão hospitalar. O Fator 3 verificado na solução fatorial encontrada neste estudo 
mostra, nas variáveis componentes do fator (V3, V4, V7, V8, V12 e V14) que a segurança da informação (o acesso só ocorre por meio de pessoas autorizadas), o armazenamento de dados de forma segura, o sigilo garantido das informações do paciente em comparação à segurança oferecida em prontuários impressos, além da prevenção de erros de transcrição de nomes de medicamentos e materiais são vantagens que contribuem com a gestão hospitalar e assistencial de maneira adequada. Sittig, Kuperman e Fiskio (1999); Costa (2001), e também Massad, Marin e Azevedo Neto (2003) já sinalizavam, na literatura, que algumas das vantagens do PEP são: a segurança dos dados, a legibilidade da informação e a atualização dos dados, entre outras. Isso corrobora os achados da AFE, especificamente do Fator 3.

Como se pôde verificar, a AFE contribuiu com a constatação de que o papel do PEP e/ou seu significado para o usuário da ferramenta é corroborado pelo que a literatura discute sobre a questão, comprovando-se três das quatro dimensões anteriormente utilizadas para a confecção do instrumento de coleta. O prontuário eletrônico contribui não somente na ótica do gestor, mas também na ótica do usuário, para o controle do trabalho, de custos e de informação da gestão e assistência hospitalar.

Outro pressuposto formulado anteriormente nesta pesquisa propunha que características biográficas e profissionais de usuários podem influenciar a percepção de que o PEP melhora seu desempenho individual. A regressão logística mostrou ( $p=0.0170$ ) que o "domínio de informática", por parte do usuário, influencia sua percepção de melhoria de desempenho individual, no uso de prontuário eletrônico.

É compreensível que o domínio de informática seja um fator influenciador da percepção de melhoria do desempenho, por parte do usuário, em relação à utilização de PEP no trabalho. Isso porque a habilidade domínio de informática é inerente e consistente com a tecnologia estudada. Ou seja, indivíduos com melhor domínio de informática manipulam com mais facilidade tecnologias de informação e comunicação. Gallouj (2002) assinala que quando a organização contempla, em seus propósitos de inovação, o conjunto de competências do usuário, as chances de sucesso da inovação podem ser mais significativas.

Com relação ao pressuposto de que na comparação entre grupos de usuários haveria diferenças significativas quanto à forma como estes percebem o prontuário eletrônico, foi possível constatar no teste $t$ (Student) realizado, com $p$-valor $<0.05$, que o hospital (ou país) a que pertence o respondente provoca diferenças significativas na forma como ele percebe essa ferramenta. Profissionais do HUF, Espanha, apresentam média 3,69 de concordância 
quanto à explicação geral do PEP. Já no HCPA, a média é menor: 3,63. Restará averiguar, em estudos futuros, o que explica tal diferença - se a diferença organizacional ou a diferença entre países - , o que pesa na diferença de percepção do PEP na ótica dos diferentes grupos (HCPA/Brasil e HUF/Espanha).

O que é possível afirmar é que as duas organizações hospitalares estão em patamar avançado de consolidação de uso de prontuário eletrônico; entretanto, em relação ao porte, a organização brasileira é maior que a organização hospitalar espanhola em número de leitos (786 no HCPA e 406 no HUF) e de funcionários (4.321 no HCPA e 1.379 no HUF). Além disso, o HCPA, no Brasil, consolidou o uso do PEP no período que compreende o final da década de 1990 e o primeiro decênio deste século XXI, enquanto o HUF, em Madri, iniciou suas atividades em 2004 com o sistema de PEP implantado. Não houve, no HUF, um período de adaptação para a mudança de prontuário impresso para prontuário eletrônico, como pode ter ocorrido no caso brasileiro.

Os resultados do teste $t$ ( $T$ Student's, $p<0.05$ ) mostraram também que o tempo de serviço no hospital interfere significativamente na forma como os respondentes percebem o significado do prontuário eletrônico para a gestão e a assistência hospitalar. Usuários com tempo de serviço igual ou menor que cinco anos apresentam média 3,71, e profissionais com tempo de serviço maior que cinco anos deslocam a média para baixo $(3,62)$ em relação ao nível de concordância com as afirmativas que definem o PEP. Para McDonald e Barnett (1990), alguns usuários podem não se acostumar com procedimentos informatizados. Por isso, os autores propõem que treinamento é medida que não pode ser excluída da discussão na adoção de prontuário eletrônico. É compreensível que pessoas com mais tempo de serviço nas organizações precisem de melhor preparo para adaptação e adoção de novas tecnologias informacionais. Talvez isso explique a média de concordância mais baixa $(3,62)$ entre sujeitos que trabalham nos hospitais pesquisados há mais de 5 anos.

Estudos futuros poderão propor a realização de análises correlacionadas visando obter, com significância estatística, uma explicação para a questão. Os resultados alcançados sugerem que há diferenças na percepção do PEP e que estas são oriundas das especificidades relativas ao perfil de usuários nas diferentes organizações hospitalares.

Propõe-se, à guisa de conclusão, que o levantamento e a observância das capacidades e competências do usuário podem contribuir com o alcance do sucesso na difusão de inovações, pois apenas "povoar réplicas de fatos consolidados" pode ser uma forma ingênua (Latour, 2000:220) de compreender a difusão da inovação. É preciso que os profissionais de saúde apresentem 
alguma sintonia com o gestor hospitalar na maneira como ambos percebem o significado e a importância do PEP.

Identificou-se, nas entrevistas iniciais da pesquisa, que os gestores são entusiastas das vantagens do emprego de prontuário eletrônico na gestão e assistência hospitalar. O usuário desse prontuário também percebe a contribuição positiva do PEP para a gestão e assistência hospitalar, porém, em nível moderado de concordância (Média 3,66, d.p. = 0.84). Essa diferença parece se explicar em razão dos papéis desses atores. A visão do gestor é global, estratégica, enquanto o executor, neste caso o usuário do prontuário eletrônico, geralmente volta suas preocupações para o desenvolvimento de atividades operacionais específicas. A visão sistêmica talvez não seja do domínio do usuário do sistema que não gerencia, mas, sim, desenvolve trabalho assistencial junto a seu paciente. A inovação administrativa em hospitais é algo complexo e desafiador enquanto objeto de análise. Esforços futuros poderiam analisar comparativamente as razões que contribuem para a diferença de percepção a respeito do prontuário eletrônico entre profissionais de saúde e gestores de hospitais.

Apresentadas as discussões e conclusões da pesquisa, é recomendável, ainda, que se estabeleça um plano de estudos futuros visando ao avanço da compreensão do fenômeno da inovação em gestão hospitalar. Assim, se propõe uma agenda futura de pesquisa.

\section{Agenda de pesquisa}

Sugerem-se estudos adicionais para o desenvolvimento teórico e prático do tema tratado nesta tese, que tenham como propósito:

a) Realizar estudo que vincule o construto aqui estudado, que define o prontuário eletrônico do paciente como ator-rede mediador de controle em organizações hospitalares, às discussões sobre satisfação e autonomia do indivíduo no trabalho;

b) Identificar fatores que expliquem as diferenças de percepção de usuários de PEP em contraste com o entusiasmo em relação à ferramenta, esboçado por gestores hospitalares;

c) Investigar razões que expliquem as diferenças de percepção do PEP quando se compara o tempo de serviço dos indivíduos em organizações hospitalares;

d) Estudar razões que expliquem as diferenças de percepção do PEP, quando comparados grupos de usuários do HCPA, no Brasil, com usuários do HUF, 
na Espanha. Será preciso esclarecer se as diferentes percepções são resultantes de fatores organizacionais (cultura organizacional, porte ou idade da organização hospitalar etc.) ou oriundas de fatores relativos aos diferentes países (origem dos sujeitos da pesquisa, cultura nacional da qual fazem parte etc.);

e) Empreender esforços empíricos para validação do instrumento de coleta elaborado e utilizado na pesquisa, contemplando 23 itens, componentes de três fatores verificados na análise fatorial exploratória realizada na pesquisa. Isto poderá, a posteriori, possibilitar a realização de análise fatorial confirmatória.

\section{Referências}

ALLISON, P.D. Logistic regression using the SAS System: theory and application. Cary, NC: SAS Institute Inc., 1999.

BIRKINSHAW, J.; HAMEL, G.; MOL, M.J. Management innovation. Academy of Management Review, v. 33, n. 4, p. 825-845, 2008.

COSTA, C.G.A. Desenvolvimento e avaliação tecnológica de um sistema de prontuário eletrônico do paciente, baseado nos paradigmas da World Wide Web e da Engenharia de Software. 2001. 288 f. Dissertação (mestrado em engenharia elétrica) — Faculdade de Engenharia Elétrica e de Computação, Universidade Estadual de Campinas, Campinas, 2001.

COYE, M.J.; HASELKORN, A.; DeMELLO, S. Remote patient management: technology-enabled innovation and evolving business models for chronic disease care. Health Affairs, v. 28, n.1, p. 126, Jan./Feb. 2009.

DAVIS, F. A technology acceptance model for empirically testing new end user information systems: theory and results. Thesis (doctoral) - Sloan School of Management, MIT Massachusetts Institute of Technology, Cambridge, 1986.

DIAS, D.S. Motivação e resistência ao uso da tecnologia da informação: um estudo entre gerentes. $R A C$, v. 4, n. 2, p. 51-66, maio/ago. 2000.

DJELLAL, F.; GALLOUJ, F. Innovation in hospitals: a survey of the literature. European Journal Health Economics, v. 8, n. 3, p. 181-193, 2007.

DJELLAL, F.; GALLOUJ, F. Mapping Innovation dynamics in hospitals. Research Policy, v. 34, n. 6, p. 817-835, 2005. 
DJELLAL, F.; GALLOUJ, F. What is innovation in services? The results of a postal survey. Éducation \& Formations, n. 59, Apr./June 2001.

FURUKAWA, M.F. et al. Adoption of health information technology for medication safety in U.S. hospitals, 2006. Health Affairs, v. 27, n. 3, p. 865-875, May/June 2008.

GALLOUJ, F. Innovation in the service economy: the new wealth of nations. Cheltenham, UK: Edward Elgar, 2002.

GIMENES, F.R.E. et al. Influência da redação da prescrição médica na administração de medicamentos em horários diferentes do prescrito. Acta Paul Enferm., v. 22, n. 4, p. 380-384, 2009.

GÜNTHER, H. Como elaborar um questionário. In: PASQUALI, L. (Org.). Instrumentos psicológicos: manual prático de elaboração. Brasília: LabPAM/Ibapp, 1999. cap. 10.

HILL, M.M.; HILL, A. A investigação por questionário. 2. ed. Lisboa: Silabo, 2002.

HOSPITAL DE CLÍNICAS DE PORTO ALEGRE. Institucional: tecnologia da Informação: sistemas de informação. Disponível em: <www.hcpa.ufrgs.br/content/ view/2380/1224/>. Acesso em: 22 fev. 2010.

HOSPITAL UNIVERSITÁRIO DE BRASÍLIA. Institucional. Disponível em: <www. hub.unb.br/Institucional/apresentacao.htm>. Acesso em: 22 fev. 2010.

HOSPITAL UNIVERSITARIO DE FUENLABRADA. El Hospital: plan estratégico: memoria 2008. Disponível em: <www.madrid.org/cs/Satellite?language=es\&pagena me=HospitalFuenlabrada\%2FPage\%2FHFLA_home > . Acesso em: 22 fev. 2010.

LATOUR, B. A esperança de Pandora. São Paulo: Edusc, 2001.

LATOUR, B. Ciência em ação. São Paulo: Editora UnesP, 2000.

LATOUR, B. Políticas da natureza. Bauru: Edusc, 2004.

MASSAD, E.; MARIN, H.F.; AZEVEDO NETO, R.S. O prontuário eletrônico do paciente na assistência, informação e conhecimento médico. Colaborador Antonio Carlos Onofre Lira. São Paulo: H. de F. Marin, 2003.

McDONALD, C.J., BARNETT, G.O. Medical-record systems. In: SHORTLIFFE, E.H.; PERREAULT, L.E. (Ed.). Medical informatics: computer applications in health care. New York: Addison-Wesley, 1990.

MENACHEMI, N. et al. Hospital adoption of information technologies and improved patient safety: a study of 98 hospitals in Florida. Journal of Healthcare Management, v. 52, n. 6, p. 398, Nov./Dec. 2007. 
MURTEIRA, B. Análise exploratória de dados: estatística descritiva. Lisboa: McGrawHill, 1993.

PEREZ, G.; ZWICKER, R. Fatores determinantes da adoção de sistemas de informação na área de saúde: um estudo sobre o prontuário médico eletrônico. Revista de Administração da Mackenzie - RAM, São Paulo, v. 11, n. 1, p. 174-200, jan./fev. 2010.

ROGERS, E. M. Diffusion of innovations. 4th. ed. New York: The Free Press, 1995.

RUBIN, D.B. Multiple imputation for nonresponsive in surveys. USA: Wiley/IEEE, 2004.

SILVA, F.G.; TAVARES-NETO, J. Avaliação dos prontuários médicos de hospitais de ensino do Brasil. Revista Brasileira de Educação Médica, v. 31, n. 2, p. 113-126, 2007.

SITTIG, D.F., KUPERMAN, G.J., FISKIO, J. Evaluating physician satisfaction regarding user interactions with an electronic medical record system. In: ANNUAL SYMPOSIUM PROCEEDINGS ARCHIVE. Proc. Amia symp. 1999. p. 400-404. Disponível em: <www.ncbi.nlm.nih.gov/pmc/articles/PMC2232602/pdf/procamiasymp00004-0437.pdf>. Acesso em: 22 fev. 2010.

SKITKA, L.J.; SARGIS, E.G. The internet as psychological laboratory. Annual Review of Psychology, v. 57, p. 529-555, 2006.

TABACHNICK, B.G.; FIDELL, L.S. Using multivariate statistics. Needham Heights: Allyn and Bacon, 2001.

VAN de VEN, A.H.; ANGLE, H.L. An introduction to the Minnesota innovation research program. In: VAN de VEN, A.H.; ANGLE, H.L.; POOLE, M.S. Research on the Management of Innovation. New York: Oxford University Press, 2000. 